Paper ID \#18838

\title{
Enhancing Communication with Students with a Teaching Method Based on Topical Guide Objectives
}

\author{
Dr. Wenbing Zhao, Cleveland State University
}

Dr. Zhao is a Full Professor at the Department of Electrical Engineering and Computer Science, Cleveland State University (CSU). He earned his Ph.D. at University of California, Santa Barbara in 2002. Dr. Zhao has a Bachelor of Science degree in Physics in 1990, and a Master of Science degree in Physics in 1993, both at Peking University, Beijing, China. Dr. Zhao also received a Master of Science degree in Electrical and Computer Engineering in 1998 at University of California, Santa Barbara. Dr. Zhao joined CSU faculty in 2004. He is currently serving as the director of the Master of Science in Electrical Engineering, and the Chair of the Graduate Program Committee in the Department of EECS, the ABET coordinator for the BS in Computer Science Program, and a member of the faculty senate at CSU. Dr. Zhao has authored a research monograph titled: "Building Dependable Distributed Systems" published by Scrivener Publishing, an imprint of John Wiley and Sons. Furthermore, Dr. Zhao published over 150 peer-reviewed papers on fault tolerant and dependable systems (three of them won the best paper award), computer vision and motion analysis, physics, and education. Dr. Zhao's research is supported in part by the US National Science Foundation, the US Department of Transportation, Ohio State Bureau of Workers' Compensation, and by Cleveland State University. Dr. Zhao has served on the organizing committee and the technical program committee for numerous international conferences. Dr. Zhao is an Associate Editor for IEEE Access, an Academic Editor for PeerJ Computer Science, and is a member of the editorial board for International Journal of Parallel Emergent and Distributed Systems, International Journal of Distributed Systems and Technologies, International Journal of Performability Engineering, International Journal of Handheld Computing Research. Dr. Zhao is a senior member of IEEE.

\section{Dr. Xiongyi Liu, Cleveland State University}

Xiongyi Liu is an Associate Professor in the Department of Curriculum and Foundations at Cleveland State University, USA. She obtained her Ph.D. in Educational Psychology from University of Nebraska, Lincoln, USA. Her research interests include technology-facilitated teaching and learning, self-regulation, and assessment and evaluation. Her expertise in research methodology has led her to serve as evaluator of multiple federal and state funded projects for preparing students of various levels for career paths in Science, Technology, Engineering, and math (STEM). Dr. Liu has been actively involved in academic community by publishing in peer-reviewed journals such as Contemporary Educational Psychology and British Journal of Educational Technology and regularly presenting at international and national conferences such as American Educational Research Association (AERA) and E-Learn.

\section{Prof. Chaomin Luo, University of Detroit Mercy}

Dr. Chaomin Luo received his Ph.D. in Department of Electrical and Computer Engineering at University of Waterloo, Canada in 2008, where he was awarded Postgraduate Scholarship (PGS) from the Natural Sciences and Engineering Research Council (NSERC) of Canada; received the Best Student Paper Presentation Award at the SWORD'2007 Conference, earned his M.Sc. in Engineering Systems and Computing at University of Guelph, Canada, and his B.Eng. degree in Radio Engineering from Southeast University, China. He is currently an Associate Professor, Department of Electrical and Computer Engineering, at University of Detroit Mercy, Michigan, USA. He was awarded Faculty Research Awards in 2009, 2010, 2014, 2015, and 2016 at University of Detroit Mercy, Michigan, USA. His research interests include engineering education, robotics and automation, control, autonomous systems, computational intelligence and machine learning.

Dr. Luo was the General Co-Chair of the 1st IEEE International Workshop on Computational Intelligence in Smart Technologies (IEEE-CIST 2015), and Journal Special Issues Chair, IEEE 2016 International Conference on Smart Technologies (IEEE-SmarTech), USA. He was the Publicity Chair in the 2011 IEEE International Conference on Automation and Logistics. He was on the Conference Committee in the 2012 International Conference on Information and Automation and International Symposium on Biomedical 
Engineering and also the Publicity Chair in the 2012 IEEE International Conference on Automation and Logistics. Also, he was Chair and Vice Chair of IEEE SEM - Computational Intelligence Chapter and is currently a Chair of IEEE SEM - Computational Intelligence Chapter and Chair of Education Committee of IEEE SEM.

Dr. Luo serves as the Editorial Board Member of International Journal of Complex Systems - Computing, Sensing and Control; Associate Editor of International journal of Robotics and Automation (IJRA); and Associate Editor of International Journal of Swarm Intelligence Research (IJSIR). He has organized and chaired several special sessions on topics of Intelligent Vehicle Systems and Bio-inspired Intelligence in IEEE reputed international conferences such as IEEE-IJCNN, IEEE-SSCI, etc. He was the Panelist in the Department of Defense, USA, 2015-2016, 2016-2017 NDSEG Fellowship program, and National Science Foundation, USA, GRFP program, 2016-2017.

Prof. Xiong Luo, University of Science and Technology, Beijing, and Beijing Key Laboratory of Knowledge Engineering for Materials Science

Xiong Luo received the Ph.D. degree from Central South University, China, in 2004. He currently works as a Professor in the School of Computer and Communication Engineering, University of Science and Technology Beijing, China. His current research interests include machine learning, cloud computing, and computational intelligence. He has published extensively in his areas of interest in journals, such as the Future Generation Computer Systems, Computer Networks, IEEE Access, and Personal and Ubiquitous Computing. 


\section{Enhancing Communication with Students Using a Teaching Method Based on Topical Guide Objectives}

\section{Introduction}

In this paper, we report a case study on employing and adapting a pedagogy based on topical guide objectives (TGOs) [1] in a senior-level undergraduate computing engineering course. According to this method, course materials are divided into a list of TGOs. Homework assignments are assigned to students at the end of every lecture. The assignments are designed explicitly around the TGOs that have been covered by each lecture. Each TGO consists of a learning objective, a set of key-points and basic concepts, correlation among them, and one or more exercise problems.

Typically, engineering/science homework is in the form of a set of problems for students to solve. The drawback of this approach is that students often get buried in the technical details and forget about the key points and concepts taught in the lectures. This new form of assignment encourages students to focus on key-points and concepts they learned in the lectures, and learn how to apply them to solve complicated problems. Furthermore, this teaching method informs students of which concepts are fundamentally important. It helps students understand the wording used on quizzes and exams. It also helps build up a positive relationship between students and the instructor such that students could focus on learning instead of testing.

\section{The TGO Teaching Method}

This method was first introduced by Dr. Mattew Morrison at the University of South Florida [1]. It was inspired by the instruction provided to enlisted Nuclear Reactor Operators in the US Navy, which focuses on the basic concepts and technical details during the training of electronics technicians, electricians, and machinist. A similar topical guide based method was independently developed for medical teaching in 2009 [2].

The TGO method contains primarily two elements: (1) inform students about what course materials are important; (2) demonstrate that students have grasped the fundamental course materials. The first element is accomplished via the definition of a set of key concepts and/or equations (referred to as topical guide objectives). The second element is done via example problems where students must use the concepts in this topic to solve. Students will gain problem solving skills in the second element. According to the TGO method, students are required to complete daily homework assignments, which consist of the above two elements: topical guide objectives for students to study, and example problems for students to solve.

According to the TGO method, quotes from people who have make significant contributions to the concept are provided to highlight the importance of the concept. Furthermore, students may also be required to research about the innovators for important topics. These two exercises help students be aware of contemporary issues.

When employing this TGO-based teaching method, we made some modifications over the original method. Figure 1 shows an example TGO homework assignment that we used in the 
Computer Networks course. In the homework assignments, for each TGO, only the concept names are given and students are asked to elaborate them in their own words as part of the homework. This would force them to learn the concepts and gain the ability to recite/paraphrase them. We decided to omit the quotes and writing assignment about innovators. We do not perceive that they will enhance student learning.

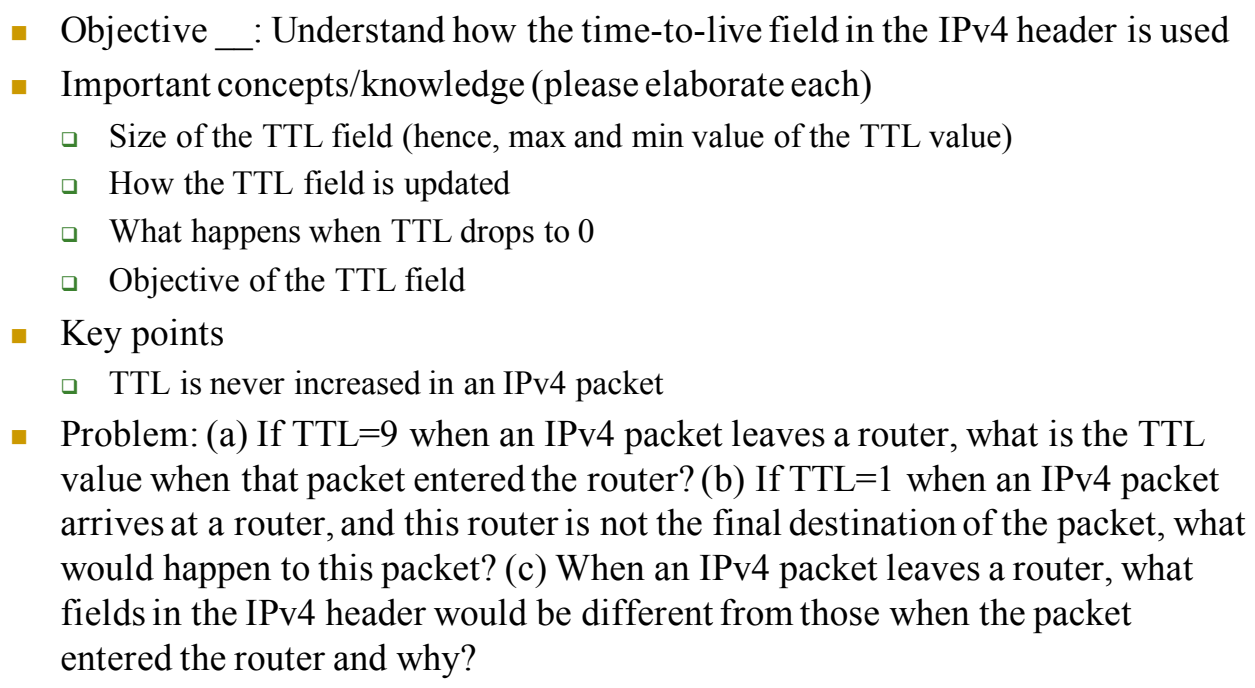

Figure 1. An example TGO homework assignment for Computer Networks.

Note that the example problems are not limited to the concepts in the current objective. It is inevitable due to the hierarchy of knowledge.

Next, we discuss the pros and cons of the TGO method, and how the TGO method can be improved to mitigate the cons. This new form of assignments encourages students to focus on key points and concepts they learned in each lecture, and learn how to apply them to solve complicated problems. Furthermore, this approach informs students which concepts are fundamentally important, also help students understand the wording used on quizzes and exams. Hence, it helps build a positive relationship between students and the instructor. This method helps students to focus on learning instead of testing. In the traditional approach, either a student would have to study carefully for every single concept and keypoint for an exam, which might not be practical considering that many undergraduate students take 5-6 courses in each semester, or take his/her chances. Students typically have no idea what subjects are important for a test. Hence, it is not surprising that many students feel that their teachers are deliberately tricking them during exams (they might have focused on one subject while another subject was tested).

The strength of the TGO method, which focuses on each objective, can also become its main disadvantage of the TGO method all homework assignments are TGO-based as described above, and all example problems are limited to individual objectives. When implementing the TGOmethod, in addition to basic example problems, one or more complex problems can be designed for students to work on. Furthermore, periodically, TGO-style comprehensive homework assignments can be given, where the relationship among a group of objectives is given, together with example problems that encompass these objectives. 
We should note that the name of TGO, that is, topical guide objectives, might give a wrong impression that the TGO-method only teaches students basic concepts. The use of example problems clearly shows that this is not the case. Even if the original TGO-method is used as it was proposed, it is inevitable for some example problems to involve concepts in two or more objectives due to the natural hierarchy of objectives. If TGO-style comprehensive homework assignments are provided periodically, this concern could be further addressed.

Another concern for the TGO-based teaching method is that the TGO-style homework assignments provide very limited opportunity for students to learn to be creative and to solve complex problems. Indeed, this is inevitable. In fact, daily homework assignments are not the most appropriate tool for this purpose. The term project is where students are asked to engage in major design activities and complex problem solving. The TGO-based teaching method not only does not exclude the use of term projects, but it prepares students to excel in their term projects also. Without having a solid understanding of fundamental concepts, it is unrealistic to expect students to be able to solve complex problems.

\section{A Case Study of Using the TGO-based Teaching Method}

We started to use the TGO-based teaching in the middle of a semester in a Computer Networks course. The decision was triggered by the extremely poor performance in the first two exams (there are four exams in the course). The course is a core course for Computer Engineering majors. We have taught the course multiple times and the student performance was decent and relatively stable across different sessions. In previous offerings, only Computer Engineering majors have enrolled in the course. However, due to a recent (inappropriate) change in the prerequisite, Electrical Engineering students were able to enroll in the course. This was compounded by the sudden increase in the enrollment of Computer Engineering students. The size of the class was usually between 10 to 15 . In this class, however, there were 23 Computer Engineering students and 16 Electrical Engineering majors. It was evident that the preparation of the students was poorer than before, especially for Electrical Engineering students.

The average of the first exam for Computer Engineering students was only 65 (out of 100), and the average of the second exam for Computer Engineering students was much lower at 50 (out of 100). Most alarmingly, the average of the first exam for Electrical Engineering students was 44 and the average of the second exam for Electrical Engineering students was an astoundingly low 20 (out of 100). Perhaps most disturbing of all was that 8 students received no credit for the second exam (7 of them were Electrical Engineering students). This was unprecedented.

We immediately designed and administered a survey after the second exam to find out what was going on and solicited suggestions on how to move forward. The survey has 19 questions and we collected valid inputs from 33 students. The survey form is shown in Figure 2. The survey result is illustrated in Figure 3. The answers to some of the questions were quite surprising to us. All students except 3 disagreed or strongly disagreed that their poor performance was due to lack of preparation, and most students claimed that they had spent substantial amount time outside the class to work on homework assignments, labs, and to prepare for the exams. Their answers to other questions are consistent with our expectations. Most of them thought the exams are too 
hard and tricky. Similarly, they thought the instructor was trying to cover too many topics. They would like to see more examples and engage in more discussions. Furthermore, they would like the class to be more interactive.

\section{Computer Networks: Midterm Survey}

First, I'd like to know how you studied for the course and why a portion of students performed very poorly in the first two exams. Please do not make any selection if you think an item is irrelevant to you.

\begin{tabular}{|c|c|c|c|c|c|}
\hline $\begin{array}{l}\text { Q1. I could not follow the lectures } \\
\text { due to lack of preparation }\end{array}$ & $\begin{array}{c}\text { Strongly } \\
\text { agree }\end{array}$ & 口 $\quad$ Agree & Neutral & $\square$ Disagree & $\begin{array}{r}\text { Strongly } \\
\text { disagree }\end{array}$ \\
\hline $\begin{array}{l}\text { Q2. I spent } \quad \text { hours per week on } \\
\text { EEC484 beyond the class time }\end{array}$ & $\begin{array}{ll}\square & 0\end{array}$ & $\square$ & $\square$ & 口 3 & 口 $\geq 4$ \\
\hline Q3. I spent _ hours for each exam & $\begin{array}{ll} & 0\end{array}$ & $\square$ & $\square$ & $\begin{array}{ll} & 3\end{array}$ & $\square \geq 4$ \\
\hline $\begin{array}{l}\text { Q4. I did group study regularly for } \\
\text { EEC484 }\end{array}$ & 口 Yes & 口 No & & & \\
\hline $\begin{array}{l}\text { Q5. I have worked on and submitted } \\
\text { all homework }\end{array}$ & 口 Yes & 口 No & & & \\
\hline $\begin{array}{l}\text { Q6. I have worked on and submitted } \\
\text { reports on all three labs }\end{array}$ & $\square \quad$ Yes & No & & & \\
\hline $\begin{array}{l}\text { Q7. I spend } \quad \text { hours on each } \\
\text { homework on average }\end{array}$ & $\square$ & $\square$ & $\square$ & $\square$ & $\square \geq 4$ \\
\hline $\begin{array}{l}\text { Q8. I spent __ hours on each lab } \\
\text { average }\end{array}$ & $\square$ & $\square$ & $\square$ & $\square$ & $\square \quad \geq 4$ \\
\hline Q9. The exam problems are too hard & $\begin{array}{c}\text { Strongly } \\
\text { agree }\end{array}$ & $\square \quad$ Agree & Neutral & $\square$ Disagree & $\begin{array}{r}\text { Strongly } \\
\text { disagree }\end{array}$ \\
\hline $\begin{array}{l}\text { Q10. The exam problems are too } \\
\text { tricky }\end{array}$ & $\begin{array}{c}\begin{array}{c}\text { Strongly } \\
\text { agree }\end{array} \\
\end{array}$ & 口 Agree & Deutral & $\square$ Disagree & $\begin{array}{r}\text { Strongly } \\
\text { disagree }\end{array}$ \\
\hline Q11. I attended all lectures except & & $\square$ & $\square$ & $\square$ & $0 \geq 4$ \\
\hline $\begin{array}{l}\text { Q12. I attended all discussion sessions } \\
\text { except }\end{array}$ & $\square$ & $\square$ & $\square$ & & \\
\hline
\end{tabular}

Next, I'd like your opinion about my teaching and how we move forward

\begin{tabular}{l|c|c|c|c|c}
\hline & $\begin{array}{c}\text { Strongly } \\
\text { Agree }\end{array}$ & Agree & Neutral & Disagree & $\begin{array}{c}\text { Strongly } \\
\text { Disagree }\end{array}$ \\
\hline Q13. The lecture covers too many topics & $\square$ & $\square$ & $\square$ & $\square$ & $\square$ \\
\hline $\begin{array}{l}\text { Q14. The homework is helpful in learning and } \\
\text { preparing for the exam }\end{array}$ & $\square$ & $\square$ & $\square$ & $\square$ & $\square$ \\
\hline $\begin{array}{l}\text { Q15. The labs are helpful in learning and } \\
\text { preparing for the exam }\end{array}$ & $\square$ & $\square$ & $\square$ & $\square$ & $\square$ \\
\hline $\begin{array}{l}\text { Q16. I would like to see more examples and } \\
\text { discussions }\end{array}$ & $\square$ & $\square$ & $\square$ & $\square$ & $\square$ \\
\hline $\begin{array}{l}\text { Q17. I would like the lectures to be more } \\
\text { interactive }\end{array}$ & $\square$ & $\square$ & $\square$ & $\square$ & $\square$ \\
\hline Q18. The grading for the exams are too harsh & $\square$ & $\square$ & $\square$ & $\square$ & $\square$ \\
\hline $\begin{array}{l}\text { Q19. I would like to see more extra credit } \\
\text { opportunities }\end{array}$ & $\square$ & $\square$ & $\square$ & $\square$ & $\square$ \\
\hline
\end{tabular}

Q20. Finally, please tell me a list of subjects you would like to spend more time on:

Figure 2. Survey form used to receive feedback from students.

In the survey, we also designated space for students to write their comments and suggestions. The most notable comment was that the homework assignments and lecture notes were not helpful to prepare them to take the exams. Even though the instructor had repeatedly told the class what to focus on and even how an example given in a class might be transformed for the upcoming exam orally, it was clearly not sufficient. The large enrollment (39) presumably made 
students harder to catch the hints given by the instructor. The survey result shows that there was a severe communication problem between the instructor and the students with respect to what will tested.

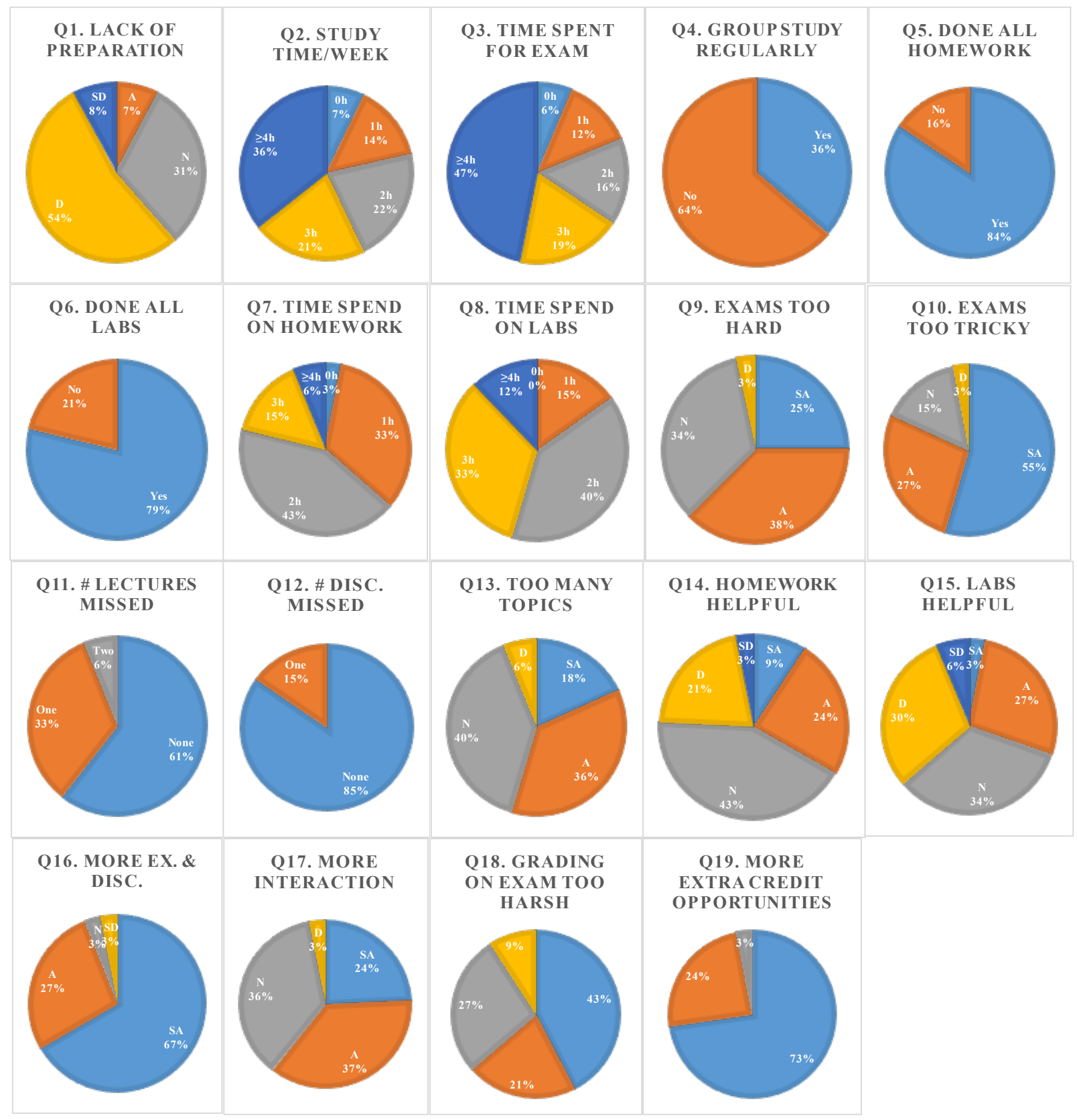

Figure 3. The first survey result.

Soon after the survey, we learned the TGO teaching method and employed it to improve communication with students (with the modifications outlined previously). It is apparent that oral communication is insufficient and some key information must be presented to student in writing. As suggested by the TGO method, daily homework assignment was issued and collected at the beginning of each lecture. In addition, during the discussion session prior to each exam, the 
relationship between different objectives and the concepts therein are discussed, with more comprehensive example problems elaborated to students.

We used the TGO method to teach the second half of the computer networks course on the network and datalink layers. We break done the network layer into seven topics, and the datalink layer into nine topics.

The network layer topics include:

- Objective 1: Able to compute the forwarding table using the link state routing method.

○ Important concepts/knowledge: Computation objective, and Information needed for the computation; Dijkstra's Algorithm; Shortest-path tree

- Key points: Never replace a temporarily labeled route by another route of longer or the same distance!

- Objective 2: Able to compute the forwarding table using the distance vector routing method.

○ Important concepts/knowledge: Computation objective; Information exchanged between neighboring nodes; Algorithm used to compute/update forwarding table

○ Key points: Never compute/update the cost/outgoing link for the entry corresponding to the router itself!

- Objective 3: Understand the issues with the distance vector routing method.

○ Important concepts/knowledge: The count-to-infinity issue; The fundamental reason for the count-to-infinity issue

- Objective 4: Understand how the time-to-live field in the IPv4 header is used.

- Important concepts/knowledge: Size of the TTL field (hence, max and min value of the TTL value); How the TTL field is updated; What happens when TTL drops to 0 ; Objective of the TTL field

- Key points: TTL is never increased in an IPv4 packet

- Objective 5: Understand the classful IPv4 addressing.

○ Important concepts/knowledge: Definition of class A, class B, and class C; IPv4 dotted decimal notation; Special IPv4 addresses; IPv4 subnetting: why and how it is accomplished; Issues with classful addressing

- Objective 6: Understand Classless InterDomain Routing (CIDR).

- Important concepts/knowledge: Rules for CIDR address allocation regarding the size of the block and the beginning of the address; What it means by "an address falls on the boundary of the block size", and how to determine it? Notation for a CIDR network in w.x.y.z/s format; How to calculate quickly the CIDR subnet mask

- Objective 7: Understand IPv4 Network Address Translation (NAT).

○ Important concepts/knowledge: Ranges of private IPv4 addresses; Key ideas behind NAT (what the NAT box would have to do); Limitations of NAT

The datalink layer topics include:

- Objective 1: Understand Error Detection Mechanisms in Datalink Layer.

- Important concepts/knowledge: Error detection principle; Parity checking mechanism; parity bit; Cyclic redundancy check (CRC) mechanism; CRC generator; modulo 2 division 
- Objective 2: Understand Multiple Access Links and Protocols.

○ Important concepts/knowledge: Types of data links; The multiple access (MAC) problem; collision; collision recovery; Classification of MAC protocols: channel partitioning (TDMA, FDMA), random access

- Objective 3: Understand Random Access Protocols.

○ Important concepts/knowledge: CSMA; Collision detection; CSMA/CD

- Objective 4: Understand the Ethernet Frame Structure.

- Important concepts/knowledge: Preamble; MAC addresses (including special addresses); type/length field; Padding, minimum frame length

- Objective 5: Understand Collision Detection in CSMA/CD.

- Important concepts/knowledge: Minimum time to detection collision; Minimum frame length; Time slot in Ethernet

- Objective 6: Understand the Ethernet MAC sublayer protocol.

○ Important concepts/knowledge: Connectionless or connection-oriented; Reliable or unreliable data communication; Ethernet CSMA/CD algorithm; Randomized exponential backoff mechanism in Ethernet protocol

- Objective 7: Understand the Address Resolution Protocol.

O Important concepts/knowledge: ARP table; ARP request; ARP reply; ARP caching; Proxy ARP

- Objective 8: Understand DHCP protocol.

○ Important concepts/knowledge: Transport level protocol used in DHCP; DHCP message exchanges; DHCP relay

- Objective 9: Understand the switch self-learning protocol.

- Important concepts/knowledge: Difference between Hubs and Switches; How

CSMA/CD is used in switches? How a switch is made transparent to hosts?

Switch self-learning protocol; How do switches work together when they are interconnected together?

We saw significant improvement in student performance in the following two exams. For exam 3 , the average for Computer Engineering students was improved to 76 (out of 100), and the average for EE students was improved to 69. For exam 4, the average for Computer Engineering students was 74 (out of 100), and the average for Electrical Engineering students was 59 (out of 100).

Towards the end of the semester, we administered another survey. The feedback from students was overwhelmingly positive on the new teaching method we experimented, although a few students did complain that the homework load was too high, which is inevitable. The second survey was based on the first one with slight changes in a few questions. The total number of questions has been reduced to 19 from 20. Some questions in the first survey were eliminated because we do not anticipate different answers from students, including question 1 on their preparation for the class, question 11 on the number of lectures missed, question 12 on the number of discussion sections missed, question 15 on whether or not the lab sessions are helpful for their exams, question 19 on more extra credit opportunities. A few new questions were added into the survey, including a question on whether or not they purchased a copy of the textbook (as question 1), two questions on their project status (questions 11 and 12), and a question on their opinion about the benefit of the projects (as question 18). The wordings of some questions are also changed (questions 13, 15, 16, and 17). 
The result for the second survey is shown in Figure 4. As can be seen, the attitude of the students has been significantly improved as seen from answers for repeated questions carried over from the first survey. The most striking improvement is for question 14 on the benefit of homework. $26 \%$ students strongly agreed and $61 \%$ students agreed (a total of $87 \%$ ) that homework is helpful to prepare exams 3 and 4 . In the first survey conducted prior to using the TGO teaching method, only $9 \%$ students strongly agreed, and $24 \%$ students agreed (a total of $33 \%$ ) that homework is helpful. Furthermore, for question 9 in the second survey, only $6 \%$ of students agreed or strongly agreed that the exams are too hard, this is done from $63 \%$.

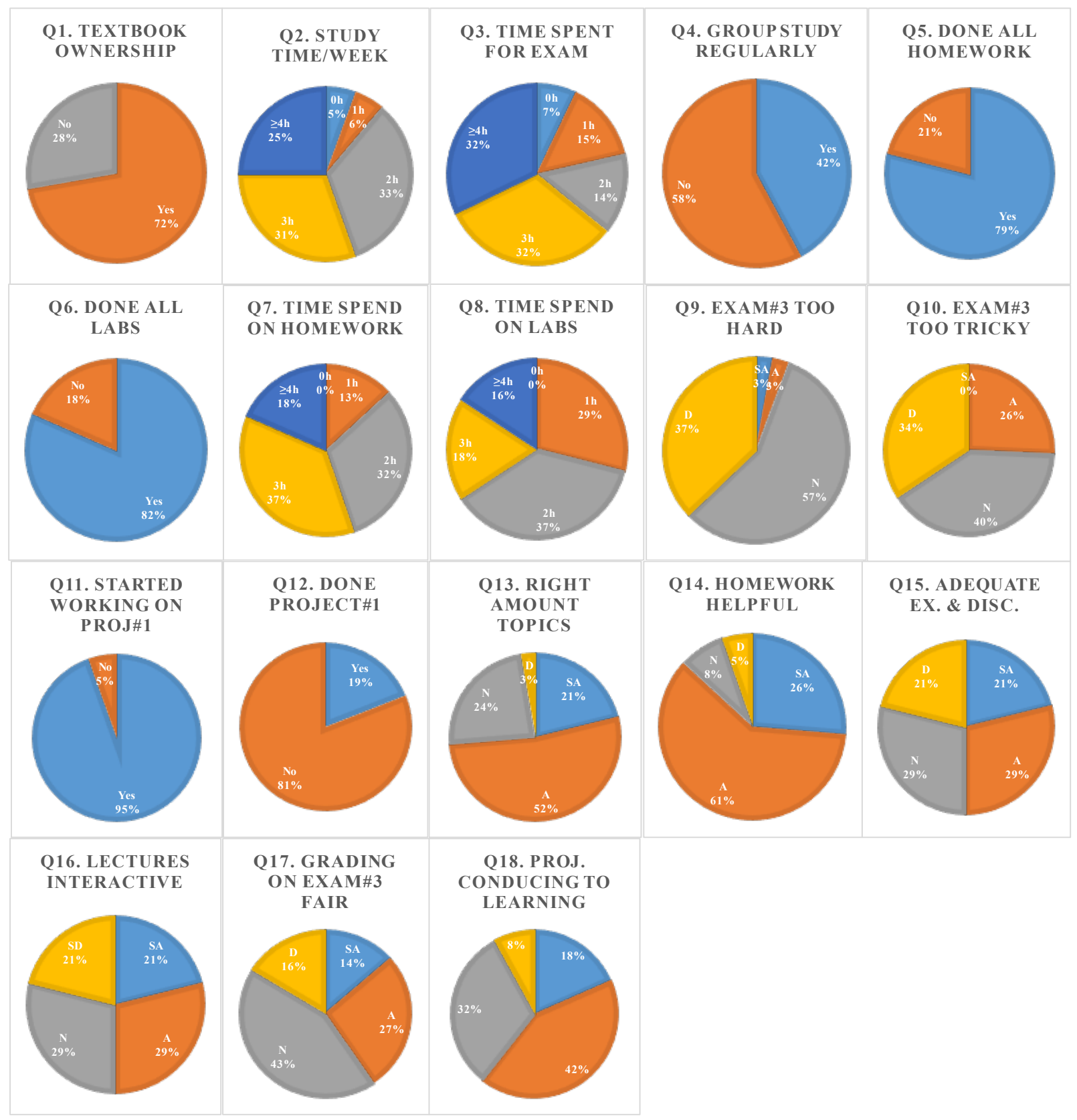

Figure 4. The second survey result. 


\section{Discussion}

We should note that the TGO teaching method does not address all the learning needs of students. We decided to employ the method to enhance the communication with students after the first survey reviewed the communication issue. The result of the second survey and the student performance on the last two exams confirmed the value of the TGO teaching method in improving the communication between students and the instructor. Another benefit of the TGO teaching method, although not unique to this method, is the constant pressure applied to students in the form of daily assignments. We find the TGO teaching method is particularly effective in helping students who are less prepared and less motivated.

The TGO teaching method can and should be used in conjunction with other teaching methods, such as active learning [3], flipped classroom [4], project-based teaching [5-9], theme-based teaching and learning approach [10], problem-based teaching methods [11,12], and experiential learning [13-15], to maximum student learning. For example, the TGO method does not address how a lecture is conducted at all. Our surveys show that students want the lectures to be more interactive, and they want to see more in-class excises and discussions.

\section{References}

1. Morrison, M. (2015). Topical Guide Objectives: A Teaching Method for Encouraging Excellence in Undergraduate STEM Student Performance. In Proceedings of the IEEE Integrated STEM Education Conference, (pp. 38-43). IEEE.

2. Gary, J., Mires Peter, W., Howie, R. M., Harden, G.A. (1998). A 'Topical' Approach to Planned Teaching and Using a Topic-Based Study Guide. Medical Teacher, 20(5), pp.438441.

3. Bonwell, C.; Eison, J. 1991. Active Learning: Creating Excitement in the Classroom. AEHEERIC Higher Education Report No. 1. Washington, D.C.: Jossey-Bass. ISBN 1-878380-08-7.

4. Bergmann, Jonathan and Sams, Aaron. (2012). Flip Your Classroom: Reach Every Student in Every Class Every Day. International Society for Technology in Education.

5. Hitt, J., \& Boettner, D., \& Suhr, S., \& Dillon, J. (2009, June), Project Based Teaching of Engineering Design Paper presented at 2009 Annual Conference \& Exposition, Austin, Texas. https://peer.asee.org/5532.

6. Constans, E., \& Kadlowec, J. (2011, June), Using a Project-Based Learning Approach to Teach Mechanical Design to First-Year Engineering Students Paper presented at 2011 ASEE Annual Conference \& Exposition, Vancouver, BC. https://peer.asee.org/18848.

7. Khorbotly, S. (2015, June), A Project-based Learning Approach to Teaching Computer Vision at the Undergraduate Level Paper presented at 2015 ASEE Annual Conference \& Exposition, Seattle, Washington. 10.18260/p.23432.

8. Zhao, W. (2015, March). Enriching engineering curricula with a course on cutting-edge computer technologies. In Proceedings of the IEEE Integrated STEM Education Conference (pp. 44-48). IEEE. 
9. Luo, C., Li, X., Wang, J., \& Zhao, W. (2015, December). Enhancement of electrical engineering education by a mentoring scheme. In Proceedings of the IEEE International Conference on Teaching, Assessment, and Learning for Engineering (pp. 72-76). IEEE.

10. Salimian, M., \& Mahmud, Y., \& Ransom, A. L. (2013, June), Theme-based Teaching /Learning: A New Approach in Teaching Manufacturing Processes Paper presented at 2013 ASEE Annual Conference \& Exposition, Atlanta, Georgia. https://peer.asee.org/22630.

11. Ocon, R. (2012, June), Teaching Creative Thinking Using Problem-based Learning Paper presented at 2012 ASEE Annual Conference \& Exposition, San Antonio, Texas. https://peer.asee.org/22002.

12. Narayanan, M. (2010, June), Assessment of Problem Based Learning Paper presented at 2010 Annual Conference \& Exposition, Louisville, Kentucky. https://peer.asee.org/15638.

13. Kolb, D. A. (2014). Experiential learning: Experience as the source of learning and development. FT press.

14. Kolb, A. Y., \& Kolb, D. A. (2005). Learning styles and learning spaces: Enhancing experiential learning in higher education. Academy of management learning \& education, $4(2), 193-212$.

15. Zhao, W. (2017). Enhancing Undergraduate Research Experience with Cutting Edge Technologies. International Journal of Information and Education Technology, 7(7), 495501. 